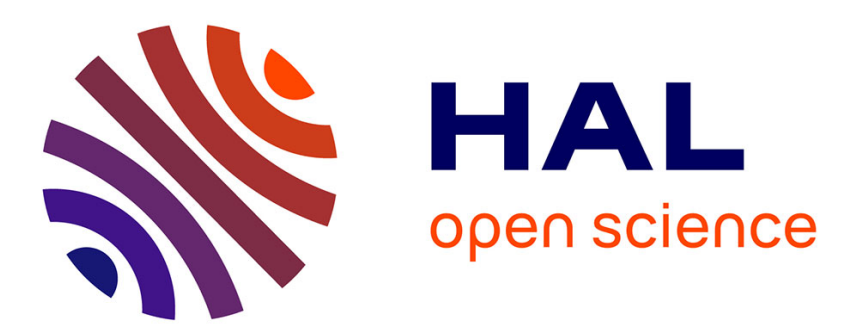

\title{
Depolarizing differential Mueller matrix of homogeneous media under Gaussian fluctuation hypothesis
}

Vincent Devlaminck

\section{To cite this version:}

Vincent Devlaminck. Depolarizing differential Mueller matrix of homogeneous media under Gaussian fluctuation hypothesis. Journal of the Optical Society of America. A, Optics and image science, 2015, 32 (10), pp.1736-1743. hal-01187677

\section{HAL Id: hal-01187677 \\ https://hal.science/hal-01187677}

Submitted on 27 Aug 2015

HAL is a multi-disciplinary open access archive for the deposit and dissemination of scientific research documents, whether they are published or not. The documents may come from teaching and research institutions in France or abroad, or from public or private research centers.
L'archive ouverte pluridisciplinaire HAL, est destinée au dépôt et à la diffusion de documents scientifiques de niveau recherche, publiés ou non, émanant des établissements d'enseignement et de recherche français ou étrangers, des laboratoires publics ou privés. 


\title{
Depolarizing differential Mueller matrix of homogeneous media under Gaussian fluctuation hypothesis
}

\author{
Vincent Devlaminck \\ Université de Lille, CRIStAL, UMR 9189, 59650 Villeneuve d'Ascq, France \\ Corresponding author:Vincent.devlaminck@univ-lille1.fr
}

\section{INTRODUCTION}

From a seminal work of Jones [1], the differential Mueller matrix formalism was introduced by Azzam [2] in polarization optics. This formalism takes into account the continuous variation of the optical properties of a medium according to the thickness $\mathrm{z}$ of the traversed medium. It relates the Mueller matrix $\mathbf{M}(z)$ at a distance $z$ into the medium to its spatial derivative along the light propagation direction by:

$$
\frac{d \mathbf{M}(z)}{d z}=\mathbf{m}(z) \mathbf{M}(z)
$$

where $\mathbf{m}(z)$ is the differential Mueller matrix.

A medium is termed homogeneous when the differential Mueller matrix $\mathbf{m}$ is constant independent of $z$. It is worth noticing that in his paper, Azzam derived the symmetry properties of $\mathbf{m}(z)$ matrix only for non-depolarizing media. More recently, Ossikovski [3] and Ortega-Quijano \& al. [4-5] have proposed a generalization of this differential matrix formalism to depolarizing Mueller matrices. They show that the logarithm of $\mathbf{M}(z)$ can always be written as:

$$
\mathbf{L}(z)=\log [\mathbf{M}(z)]=\left[\begin{array}{cccc}
P_{0}(z) & P_{4}(z)+D_{4}(z) & P_{5}(z)+D_{5}(z) & P_{6}(z)+D_{6}(z) \\
P_{4}(z)-D_{4}(z) & P_{0}(z)-D_{7}(z) & D_{3}(z)+P_{3}(z) & D_{2}(z)-P_{2}(z) \\
P_{5}(z)-D_{5}(z) & D_{3}(z)-P_{3}(z) & P_{0}(z)-D_{8}(z) & D_{1}(z)+P_{1}(z) \\
P_{6}(z)-D_{6}(z) & D_{2}(z)+P_{2}(z) & D_{1}(z)-P_{1}(z) & P_{0}(z)-D_{9}(z)
\end{array}\right]
$$

where $P_{0}(z)$ is the isotropic absorption; $P_{1-6}(z)$ are the elementary polarization properties: linear, $\pm 45^{\circ}$ and circular birefringence $\left(P_{1-3}\right)$ and dichroism $\left(P_{4-6}\right)$; the off diagonal depolarizations $D_{1-6}(z)$ are the uncertainties of the respective properties whereas the diagonal terms $D_{7-9}(z)$ represent the anisotropic depolarizations. All these properties are estimated at the distance $z$ into the medium.

The mathematical conditions that ensure the existence of such a logarithm are well known [6]. However, these conditions are not really sufficient in this present case because it is also necessary to ensure that the matrix $\mathbf{M}$ obtained from $\mathbf{m}$ is a Mueller matrix for any $z$ values. The conditions under which the logarithm of such matrix is physically well defined (meaning that $\mathbf{M}(z)$ is a physically realizable Mueller matrix for any for any $z$ values) have been already addressed [7] and a general criterion has been derived. $\mathbf{L}(z)$ can be decomposed into its Minkowski antisymmetric and symmetric components $\mathbf{L}_{m}$ and $\mathbf{L}_{u}$ related respectively to the polarization and depolarization properties of the medium. The positive semidefiniteness of a $3 \times 3$ restriction of the coherency matrix of the symmetric component ensures the physical realizability of the differential Mueller matrix. The issue of non-uniqueness of the solution was also discussed and the physical meaning of the multiplicity of possible solutions is given in [8]. 
In a recent paper [9] we extend the scope of the depolarizing Mueller calculus to parallel that established by Jones for his calculus based on the layered-medium interpretation. With this model, the matrix $\mathbf{m}(z)$ becomes a random process and Eq. (1) is now a stochastic differential equation. Considering $\mathbf{m}(z)$ as a stochastic operator involves however a sophisticated solution since the matrices $\mathbf{m}\left(z_{1}\right)$ and $\mathbf{m}\left(z_{2}\right)$ do not necessarily commute for $z_{1} \neq z_{2}$. But it is worth noting that we are actually only interested in the average value of the solution of Eq. (1) since the general Mueller matrix $\mathbf{M}(z)$ is then seen as an average value of non-depolarizing Mueller matrices (or Mueller-Jones matrices $\mathbf{M J}(z)$ associated to their differential matrices $\mathbf{m}_{\mathbf{N D}}(z)$ ) but random. In this case, Eq. (1) can be formally solved using the z-ordered operator introduced by Fox [15]:

$$
\mathbf{M}(z)=\langle\mathbf{M J}(z)\rangle=\left\langle\underset{\leftarrow}{\operatorname{Zexp}}\left[\int_{0}^{z} \mathbf{m}_{\mathrm{ND}}(\mathrm{s}) \mathrm{ds}\right]\right\rangle \mathbf{M}(0)
$$

where $Z$ is a space-ordering operator that causes the factors in a product to appear in the same sequence as in the medium of interest. It is defined by:

$$
\underset{\leftarrow}{Z}\left[\left(\int_{0}^{z} \mathbf{f}(s) d s\right)^{n}\right]=n ! \int_{0}^{z} d z_{n} \int_{0}^{z_{n}} d z_{n-1} \cdots \int_{0}^{z_{2}} d z_{1} \mathbf{f}\left(z_{\mathrm{n}}\right) \mathbf{f}\left(z_{\mathrm{n}-1}\right) \cdots \mathbf{f}\left(z_{1}\right)
$$

Giving the explicit form of the z-ordered exponential can be achieved by using a power series expansion of the exponential. The differential Mueller matrix is denoted by using $p_{\mathrm{i}}$ and $d_{\mathrm{i}}$ the $z$ differential properties:

$$
\mathbf{m}(s)=\left[\begin{array}{cccc}
p_{0}(s) & p_{4}(s)+d_{4}(s) & p_{5}(s)+d_{5}(s) & p_{6}(s)+d_{6}(s) \\
p_{4}(s)-d_{4}(s) & p_{0}(s)-d_{7}(s) & d_{3}(s)+p_{3}(s) & d_{2}(s)-p_{2}(s) \\
p_{5}(s)-d_{5}(s) & d_{3}(s)-p_{3}(s) & p_{0}(s)-d_{8}(s) & d_{1}(s)+p_{1}(s) \\
p_{6}(s)-d_{6}(s) & d_{2}(s)+p_{2}(s) & d_{1}(s)-p_{1}(s) & p_{0}(s)-d_{9}(s)
\end{array}\right]
$$

Splitting $\mathbf{m}(z)$ into its Minkowski antisymmetric and symmetric components as proposed by Ossikovski [3], leads respectively to $\mathbf{m}_{\mathbf{N D}}(z)$ and $\mathbf{m}_{\mathbf{D}}(z)$ the non-depolarizing and depolarizing parts of $\mathbf{m}(z)$. It is worth noticing that in the general case we have:

$$
\begin{aligned}
& P_{i}(z) \neq \int_{0}^{z} p_{i}(s) d s \\
& D_{i}(z) \neq \int_{0}^{z} d_{i}(s) d s
\end{aligned}
$$

This is a consequence of the presence of $Z$ the space-ordering operator in Eq. (3).

In this paper we first address the issue of the conditions of existence of a $z$-independent depolarizing differential Mueller matrix related to a homogeneous medium. Such a medium is characterized by linear changes of its non-depolarizing and depolarizing optical properties with $z$ the thickness of the medium. In the first part of this paper, we show that standard assumption of white noise is sufficient to ensure the linearity of $\log (\mathbf{M}(z))$ according to $z$. A first example of linear solution has been previously given in [9] for instance. As noted by the authors of a recent paper [10], this solution needs to be "symmetrised" because of the non-commutativity of the individual matrices associated to each layer.

However, in these two recent articles, apparently contradictory proposals have emerged. In one case |9], a physical model of fluctuating layers leads to a linear $z$ term for depolarizer part while in the other case [10] an assumption of first order approximation of $z$ leads to a quadratic $z$ term solution for the depolarizer.

Which of these solutions is the right one or, more precisely in what cases they can be applied? Also, what are we talking about when we are talking about first order in $z$ approximation from an experimental point of view? 
In the second part of this paper we clarify the general form of $\mathbf{m}(z)$ in the case where optical properties of the medium are supposed to be random white Gaussian noise-like or short correlation length processes. We show that it solves the problem of non-commutativity while maintaining a $z$-linear solution. Finally, the recently proposed approach [10] for analysis of the statistical properties related to changes in optical properties is revisited and the experimental conditions of application of these results are also specified.

\section{EXISTENCE OF A Z-LINEAR DEPOLARIZING DIFFERENTIAL MUELLER MATRIX}

In his paper [2] Azzam derived the relations between the entries of "N-matrices" introduced by Jones and $\mathbf{m}$ differential matrices for non-depolarizing media. However, the formal relation between these both matrices was formulated by Barakat [11]. From the concept of exponential versions of the Mueller-Jones matrices and properties of the Kronecker product of matrices, Barakat established the following relation:

$$
\mathbf{m}=\Lambda^{\dagger}\left(\mathrm{N} \otimes I+I \otimes N^{*}\right) \Lambda
$$

where $\uparrow$ and $*$ stand for a Hermitian and complex conjugate respectively, $\mathbf{N}$ is the logarithm of the corresponding Jones matrix and

$$
\Lambda=\frac{1}{\sqrt{2}}\left[\begin{array}{cccc}
1 & 1 & 0 & 0 \\
0 & 0 & 1 & -\mathrm{i} \\
0 & 0 & 1 & \mathrm{i} \\
1 & -1 & 0 & 0
\end{array}\right]
$$

If $A$ is an $m$-by- $n$ matrix and $B$ is a $p$-by- $q$ matrix, then the Kronecker product is the $m p$-by- $n q$ block matrix defined by:

$$
\mathbf{A} \otimes \mathbf{B}=\left[\begin{array}{ccc}
\mathrm{a}_{11} \mathbf{B} & \cdots & \mathrm{a}_{1 \mathfrak{1 \eta}} \mathbf{B} \\
\vdots & \ddots & \vdots \\
\mathrm{a}_{\mathrm{m} 1} \mathbf{B} & \cdots & \mathrm{a}_{\mathrm{mn}} \mathbf{B}
\end{array}\right]
$$

We address some hypothesis that should be verified before solving the Mueller matrix differential equation proposed by Azzam when depolarizing media is under analysis. Let us consider the very elementary case of homogeneous non-depolarizing media. Under this hypothesis, the corresponding Mueller-Jones matrix $\mathbf{M} \mathbf{J}(z)$ possesses an exponential representation $\mathbf{M} \mathbf{J}(z)=$ $\exp (z . \mathbf{m})$. Thus, for homogeneous media the macroscopic property associated to this kind of Mueller matrices is:

$$
\mathbf{M J}\left(z_{2}+z_{1}\right)=\mathbf{M J}\left(z_{2}\right) \cdot \mathbf{M} \mathbf{J}\left(z_{1}\right)
$$

In fact, the Mueller-Jones matrices inherit to the property from the corresponding Jones matrices $\mathbf{J}\left(z_{2}+z_{1}\right)=\mathbf{J}\left(z_{2}\right)$. $\mathbf{J}\left(z_{1}\right)$. This point was already stressed by Barakat [11] but without noticing that this property is only verified for homogeneous media.

Let us now consider the extension of this property to the depolarizing media. Such a medium could be characterized by its Mueller matrix. This matrix M may be considered as the Mueller matrix of an optical system exhibiting spatial, temporal or frequency heterogeneity over the area illuminated by the incident light. The light beam is shared among these elements characterized by Mueller-Jones matrices $\mathbf{M} \mathbf{J}_{\mathrm{k}}$ according to ratio $\alpha_{\mathrm{k}}$. The emerging light is consequently composed of these incoherent contributions in the same proportions and following Kim et al. [12], we define a Mueller matrix $\mathbf{M}$ as a weighted 
sum with positive weights (a convex sum) of so-called Mueller-Jones matrices also named pure Mueller matrices or nondepolarizing Mueller matrices

$$
\mathbf{M}=\sum_{k} \alpha_{k} \mathbf{M} \mathbf{J}_{k} \equiv\left\langle\mathbf{M} \mathbf{J}_{k}\right\rangle
$$

These $\mathbf{M} \mathbf{J}_{k}$ matrices are obtained by writing the equation $\mathbf{E}^{\prime}=\mathbf{J}_{k} \mathbf{E}$, mapping an input electric field vector $\mathbf{E}$ into an output electric field vector $\mathbf{E}^{\prime}$ by means of the $2 \times 2$ Jones matrix $\mathbf{J}_{k}$, in terms of a relation between the corresponding vectors of Stokes parameters.

Considering the Mueller matrix of the system as a mean value of an ensemble defined by Eq. (11), a straightforward application [12] of the relations between Jones and Mueller-Jones matrices, gives the following relation:

$$
\mathbf{M}=\Lambda^{\dagger} \mathbf{F} \Lambda \text { and } \mathbf{F}=\Lambda \mathbf{M} \Lambda^{\dagger}
$$

where $\mathbf{F}$ is defined as the average value of realizations of the Kronecker product of the $\mathbf{J}$ matrix and its conjugate $\mathbf{J}^{*}$

$$
\mathbf{F}=\left\langle\mathbf{J}_{k} \otimes \mathbf{J}_{k}^{*}\right\rangle=\left[\begin{array}{llll}
\left\langle\mathrm{j}_{\mathrm{k}, 0} \mathrm{j}_{\mathrm{k}, 0}^{*}\right\rangle & \left\langle\mathrm{j}_{\mathrm{k}, 0} \mathrm{j}_{\mathrm{k}, 1}^{*}\right\rangle & \left\langle\mathrm{j}_{\mathrm{k}, 1} \mathrm{j}_{\mathrm{k}, 0}^{*}\right\rangle & \left\langle\mathrm{j}_{\mathrm{k}, 1} \mathrm{j}_{\mathrm{k}, 1}^{*}\right\rangle \\
\left\langle\mathrm{j}_{\mathrm{k}, 0} \mathrm{j}_{\mathrm{k}, 2}^{*}\right\rangle & \left\langle\mathrm{j}_{\mathrm{k}, 0} \mathrm{j}_{\mathrm{k}, 3}^{*}\right\rangle & \left\langle\mathrm{j}_{\mathrm{k}, 1} \mathrm{j}_{\mathrm{k}, 2}^{*}\right\rangle & \left\langle\mathrm{j}_{\mathrm{k}, 1} \mathrm{j}_{\mathrm{k}, 3}^{*}\right\rangle \\
\left\langle\mathrm{j}_{\mathrm{k}, 2} \mathrm{j}_{\mathrm{k}, 0}^{*}\right\rangle & \left\langle\mathrm{j}_{\mathrm{k}, 2} \mathrm{j}_{\mathrm{k}, 1}^{*}\right\rangle & \left\langle\mathrm{j}_{\mathrm{k}, 3} \mathrm{j}_{\mathrm{k}, 0}^{*}\right\rangle & \left\langle\mathrm{j}_{\mathrm{k}, 3} \mathrm{j}_{\mathrm{k}, 1}^{*}\right\rangle \\
\left\langle\mathrm{j}_{\mathrm{k}, 2} \mathrm{j}_{\mathrm{k}, 2}^{*}\right\rangle & \left\langle\mathrm{j}_{\mathrm{k}, 2} \mathrm{j}_{\mathrm{k}, 3}^{*}\right\rangle & \left\langle\mathrm{j}_{\mathrm{k}, 3} \mathrm{j}_{\mathrm{k}, 2}^{*}\right\rangle & \left\langle\mathrm{j}_{\mathrm{k}, 3} \mathrm{j}_{\mathrm{k}, 3}^{*}\right\rangle
\end{array}\right]
$$

where $k$ designates 'noise' realizations,

Generalization of this result to functions of space, leads to the concept of random process [13]. To define this random process we assign a complex-valued matrix $\mathbf{F}(k, z)$ with independent variable $z$ to each elementary event $k$. A second order probability density function may be associated to this process and a corresponding second order joint moment or classically termed "statistical correlation" "between the two random variables $\mathbf{F}\left(k, z_{1}\right)$ and $\mathbf{F}\left(k, z_{2}\right)$ can be defined.

Given these expressions, the relevant quantities at distance $z_{1}+z_{2}$ into a depolarizing medium (as the result of a convex sum of relevant quantities associated to non-depolarizing media) are:

$$
\mathbf{M}\left(z_{2}+z_{1}\right)=\Lambda^{\dagger}\left\langle\mathbf{J}_{k}\left(z_{2}+z_{1}\right) \otimes \mathbf{J}_{k}^{*}\left(z_{2}+z_{1}\right)\right\rangle \Lambda
$$

Decomposing $\mathbf{J}_{k}\left(z_{2}+z_{1}\right)$ into a product of two matrices corresponding to the transmission from 0 to $z_{1}$ and $z_{1}$ to $z_{1}+z_{2}$ leads to:

$$
\mathbf{J}_{k}\left(z_{2}+z_{1}\right)=\mathbf{J}_{m}\left(z_{1}, z_{2}+z_{1}\right) \mathbf{J}_{l}\left(0, z_{1}\right)
$$

Where $k, l, m$ designate independent noise realizations. Employing the well known Kronecker product identity $($ A.B $) \otimes($ C.D $)=$ $(\mathbf{A} \otimes \mathbf{C})$.(B $\otimes \mathbf{D})$ after substituting $\mathbf{J}_{k}\left(z_{2}+z_{1}\right)$ in Eq. (14) gives:

$$
\mathbf{M}\left(z_{2}+z_{1}\right)=\Lambda^{\dagger}\left\langle\left[\mathbf{J}_{m}\left(z_{1}, z_{2}+z_{1}\right) \otimes \mathbf{J}_{m}^{*}\left(z_{1}, z_{2}+z_{1}\right)\right] \cdot\left[\mathbf{J}_{l}\left(0, z_{1}\right) \otimes \mathbf{J}_{l}^{*}\left(0, z_{1}\right)\right]\right\rangle \Lambda
$$


In order to achieve the separation of $\mathbf{M}\left(z_{1}+z_{2}\right)=\mathbf{M}\left(z_{2}\right) . \mathbf{M}\left(z_{1}\right)$ or more precisely (with $\Lambda \Lambda^{\dagger}=\mathbf{I}$ ):

$$
\mathbf{M}\left(z_{2}+z_{1}\right)=\Lambda^{\dagger}\left\langle\left[\mathbf{J}_{m}\left(z_{2}\right) \otimes \mathbf{J}_{m}^{*}\left(z_{2}\right)\right]\right\rangle \Lambda \cdot \Lambda^{\dagger}\left\langle\left[\mathbf{J}_{l}\left(z_{1}\right) \otimes \mathbf{J}_{l}^{*}\left(z_{1}\right)\right]\right\rangle \Lambda
$$

we just need to postulate second order stationary random processes:

$$
\left\langle\mathbf{J}_{m}\left(z_{1}, z_{2}+z_{1}\right) \otimes \mathbf{J}_{m}^{*}\left(z_{1}, z_{2}+z_{1}\right)\right\rangle=\left\langle\mathbf{J}_{m}\left(0, z_{2}\right) \otimes \mathbf{J}_{m}^{*}\left(0, z_{2}\right)\right\rangle
$$

and:

$$
\left\langle\mathbf{F}\left(k, z_{1}\right) \mathbf{F}\left(l, z_{2}\right)\right\rangle=\left\langle\mathbf{F}\left(k, z_{1}\right)\right\rangle\left\langle\mathbf{F}\left(l, z_{2}\right)\right\rangle
$$

Meaning that both physical situations of medium at distance $z_{2}$ and $z_{1}$ are uncorrelated (white noise hypothesis).

Under this un-correlated stationary process hypothesis, $\mathbf{M}(z)$ possesses an exponential representation :

$$
\mathbf{M}(z)=\exp (z \mathbf{m})=\mathbf{I}+z \mathbf{m}+\sum_{\mathbf{k}=2}^{+\infty} \frac{z^{\mathrm{k}}}{\mathrm{k} !}(\mathbf{m})^{\mathrm{k}}
$$

where $\mathbf{m}$ is the differential Mueller matrix that is constant independent of $z$.

It is worth noticing that in order for $\mathbf{M}$ to become homogeneous and the ensemble averages over $k$ and $l$ to result in the same generating differential Mueller matrix, the homogeneity of the underlying non-depolarizing matrices has to be sacrificed. This appears even more clearly if the fluctuations are related to the model that we recently proposed [9] where the medium is composed of a stack of random laminae with basic polarization properties. The random fluctuations that create the uncorrelation between different depths intrinsically make the underlying non-depolarizing matrices locally non-homogeneous but the average behavior remains homogeneous.

Nevertheless the preceding equations are formal expressions. We now derive explicit expressions of $z$-linear solution under some specific hypothesis.

\section{THE GAUSSIAN WHITE NOISE AND SHORT CORRELATION DISTANCE CASES.}

We will now assume that the birefringence and dichroïsm components fluctuations are purely random, stationary, Gaussian processes. Considering this depolarizing Mueller matrix as a mean value of an ensemble of non-depolarizing matrices leads to an expression for the Mueller matrix given by Eq. (3) where $\mathbf{M}(0)$ is assumed to be the identity matrix which is obviously not a restrictive condition.

The differential matrix $\mathbf{m}$ is parameterized as in Eq. (5) and for a non-depolarizing medium: $d_{i}=0$ and $\mathbf{m}(z)=\mathbf{m}_{\mathbf{N D}}(z)$. It is well known that $\mathbf{m}_{\mathbf{N D}}(z)$ can be written as a sum of the six generators $\mathbf{G}_{1-6}$ of $S O(3,1)$ weighted by $p_{1-6}(z)$ and $\mathbf{G}_{0}$ the identity matrix weighted by the differential isotropic absorption $p_{0}(z)$ :

$$
\mathbf{m}_{\mathrm{ND}}(z)=\sum_{\mathrm{i}=0}^{6} p_{\mathrm{i}}(z) \mathbf{G}_{\mathrm{i}}
$$

These generators and their relations are described in Appendix A of [9] for instance. 
In order to evaluate the average of the exponential of the integral of $\mathbf{m}_{\mathrm{ND}}(z)$, it is necessary to know all the moments or cumulants [13] of $\mathbf{m}_{\mathrm{ND}}(z)$. With a Gaussian scalar process hypothesis, the solution is greatly facilitated since such a process leads to an explicit determination of any order moment in terms of the first (average) and second (correlation) moments. Reformulating this property in terms of cumulant leads to say that all the cumulants of higher order than second one vanish identically.

Considering the stochastic operator $\mathbf{m}(z)\left(\mathbf{m}_{\mathrm{ND}}(z)\right.$ will be noted $\mathbf{m}(z)$ thereafter to simplify the notation) involves a more sophisticated solution. With a Gaussian process hypothesis, the $\mathbf{m}(z)$ matrix is a stochastic operator where matrix entry by matrix entry the process is Gaussian. For the estimation of the higher order moment of $\mathbf{m}(z)$, the order of the integrand factors is now important since the matrices $\mathbf{m}\left(z_{1}\right)$ and $\mathbf{m}\left(z_{2}\right)$ do not commute for $z_{1} \neq z_{2}$ in general. Using the $z$-ordered operator introduced by Fox [15] Eq. (1) can be formally solved and this formal expression is given by Eq. (3). Giving the explicit form of the z-ordered exponential can be achieved by using a power series expansion of the exponential leading to an explicit determination of any order moment. Nevertheless as demonstrated by Fox [17] the evaluation of Eq. (3) is better suited by using the z-ordered cumulants. We have:

$$
\left\langle\underset{\leftarrow}{\operatorname{Zexp}}\left(\int_{0}^{z} \mathbf{m}(s) \mathrm{d} s\right)\right\rangle=\underset{\leftarrow}{\operatorname{Zexp}}\left(\sum_{\mathrm{n}=1}^{+\infty} \int_{0}^{z} \mathbf{c}^{(\mathbf{n})}(s) \mathrm{d} s\right)
$$

where $^{(\mathrm{n})}$ stands for the cumulant of $n$ order. The integral of the first and second z-ordered cumulants are given by [14]:

$$
\begin{aligned}
& \int_{0}^{z} \mathbf{c}^{(\mathbf{1})}(s) \mathrm{d} s=\int_{0}^{z}\langle\mathbf{m}(s)\rangle \mathrm{d} s \\
& \int_{0}^{z} \mathbf{c}^{(2)}(s) \mathrm{d} s=\int_{0}^{z}\left\langle\mathbf{m}(s)^{2}\right\rangle \mathrm{d} s-\frac{1}{2} \mathrm{Z}\left[\left(\int_{0}^{z}\langle\mathbf{m}(s)\rangle \mathrm{d} s\right)^{2}\right]
\end{aligned}
$$

Even if $\mathbf{m}(z)$ is assumed to be Gaussian, the non-commutativity of this matrix for different $z$ values, annihilates the classical scalar property that all the cumulants of higher order than second cumulant are equal to zero. However there is at least one case in which this property is still valid [15]: if the stochastic processes are assumed to be white noise. Under this hypothesis, the average and correlation of the fluctuations associated to the centred processes are given by:

$$
\begin{aligned}
& \left\langle m_{i j}(z)\right\rangle=\mu_{i j} \\
& \left\langle\left(m_{i j}\left(z_{2}\right)-\mu_{i j}\right)\left(m_{k l}\left(z_{1}\right)-\mu_{k l}\right)\right\rangle=\sigma_{i j k l} \delta\left(z_{2}-z_{1}\right)
\end{aligned}
$$

where $\delta(z)$ is the Dirac delta function.

$\langle\mathbf{M}(z)\rangle$ is thus reduced to:

$$
\left\langle\leftarrow \operatorname{Zexp}\left(\int_{0}^{z} \mathbf{m}(s) \mathrm{d} s\right)\right\rangle=\underset{\leftarrow}{\operatorname{Zexp}}\left(\int_{0}^{z} \mathbf{c}^{(\mathbf{1})}(s) \mathrm{d} s+\int_{0}^{z} \mathbf{c}^{(\mathbf{2})}(s) \mathrm{d} s\right)
$$

Assuming $\mathbf{m}_{\mathbf{n d}}(z)$ defined by Eq. (21) as such Gaussian stochastic operator with: 


$$
\begin{aligned}
& \left\langle p_{i}(z)\right\rangle=\mu_{i} \\
& \left\langle\left(p_{i}\left(z_{2}\right)-\mu_{i}\right)\left(p_{j}\left(z_{1}\right)-\mu_{j}\right)\right\rangle=\sigma_{i j} \delta\left(z_{2}-z_{1}\right)
\end{aligned}
$$

where $\sigma_{\mathrm{ij}}$ denotes the entries of the covariance matrix of the centred Gaussian white noises $p_{\mathrm{i}}(z)\left(\sigma_{\mathrm{ii}}\right.$ will be denoted by $\sigma_{\mathrm{i}}^{2}$ and is the variance of the process), the following relation can be derived (See Appendix A for details of the calculation):

$$
\begin{aligned}
\left\langle\mathbf{M}_{\mathbf{n d}}(z)\right\rangle & =\underset{\leftarrow}{\operatorname{Z}} \exp \left(\int_{0}^{z} \mathbf{c}^{(\mathbf{1})}(s) \mathrm{d} s+\int_{0}^{z} \mathbf{c}^{(\mathbf{2})}(s) \mathrm{d} s\right) \\
& =\exp \left[z\left(\sum_{\mathrm{i}=0}^{6} \mu_{\mathrm{i}} \mathbf{G}_{\mathrm{i}}+\sum_{\mathrm{i}, \mathrm{j}=0}^{6} \sigma_{\mathrm{ij}} \mathbf{G}_{\mathrm{i}} \mathbf{G}_{\mathrm{j}}\right)\right] \\
& =\exp [\mathbf{L}(z)]
\end{aligned}
$$

Assuming that the stochastic processes have finite short correlation distances (meaning that for any two distances such that their difference is greater than these correlation distances, all matrix entries at these two distances are statistically independent) leads to conclude that Eq. (25) is still valid if the amplitude of the fluctuations (formally noted by $\alpha$ ) are small compared to $(\zeta)^{-1}$ the inverse of correlation distances [16]. This short correlation distances hypothesis implies that each cumulant in Eq. (22) vanishes unless the distance points $z_{\mathrm{i}}$ and $z_{\mathrm{j}}$ in Eq. (4) verify $\left|z_{\mathrm{i}}-z_{\mathrm{j}}\right| \leq \zeta$. It is worth noticing that Eq. (22) is not an expansion in power of $(\alpha . z)$ but can be considered as an expansion in power of $(\alpha . \zeta)$, each cumulant being considered as linear in $z$ (see [16], p. 388 for more details). Fox for his part has also shown [15] that the first order or linear, in $z$ property described by Eq. (27) for a correlation involving a Dirac delta function, remains true if the correlation matrix in Eq. (24) dies out on the length $\zeta$. He has shown that for $z \gg \zeta$ a cluster property of the higher cumulants applies and they all approach a linear dependence in $z$. The $Z$ ordering exponential of Eq. (22) can be replaced by a classical matrix exponential according to the relation:

$$
\underset{\leftarrow}{Z} \exp \left(\sum_{\mathrm{n}=1}^{+\infty} \int_{0}^{z} \mathbf{c}^{(\mathbf{n})}(s) \mathrm{d} s\right) \underset{\mathrm{z} \gg \zeta}{\rightarrow} \exp \left(\sum_{\mathrm{n}=1}^{+\infty} z \mathbf{M}^{(\mathrm{n})}\right)
$$

where the $\mathbf{M}^{(\mathrm{n})}$ are $z$-independent matrices (see [15] for more details on these matrices). As noted by Fox [15], the infinite sum on $\mathbf{M}^{(\mathrm{n})}$ can be often well approximated by its first two terms. One then finds an expression of $\mathbf{L}(z)$ corresponding to the case of white noise like fluctuation but only for $z$ values much larger than $\zeta$ and a sufficient decay ensured by the condition $\alpha . \zeta \ll 1$.

Reformulating Eq. (27) in order to decompose $\mathbf{L}(z)$ into its Minkowski antisymmetric $\left(\mathbf{L}_{\mathrm{m}}\right)$ and symmetric $\left(\mathbf{L}_{\mathrm{u}}\right)$ components as proposed by Ossikovski [3] can be achieved by using the following relation:

$$
\mathbf{G}_{\mathrm{i}} \mathbf{G}_{\mathrm{j}}=\frac{1}{2}\left\{\mathbf{G}_{\mathrm{i}}, \mathbf{G}_{\mathrm{j}}\right\}+\frac{1}{2}\left[\mathbf{G}_{\mathrm{i}}, \mathbf{G}_{\mathrm{j}}\right]
$$

where $[\mathbf{A}, \mathbf{B}]=\mathbf{A B}-\mathbf{B A}$ is the commutator operator and $\{\mathbf{A}, \mathbf{B}\}=\mathbf{A B}+\mathbf{B A}$ is the anticommutator operator. Explicit expressions of these operators for $\mathbf{G}_{\mathbf{i}}$ matrices can be found in [9] as well as details of an equivalent calculation to bring up the symmetric and antisymmetric parts of $\mathbf{L}(z)$ defined by Eq.(2). According to the expressions of those operators and Eqs. (27) and (29) the nondepolarizing part $\mathbf{L}_{\mathrm{m}}(z)$ and depolarizing part $\mathbf{L}_{\mathrm{u}}(z)$ of $\mathbf{m}(z)$ are thus given by: 


$$
\begin{gathered}
\mathbf{L}_{\mathrm{u}}(z)=z \times\left[\begin{array}{cccc}
\sigma_{4}^{2}+\sigma_{5}^{2}+\sigma_{6}^{2} & -\sigma_{26}+\sigma_{35} & -\sigma_{34}+\sigma_{16} & -\sigma_{15}+\sigma_{24} \\
\sigma_{26}-\sigma_{35} & \sigma_{4}^{2}-\sigma_{2}^{2}-\sigma_{3}^{2} & \sigma_{12}+\sigma_{45} & \sigma_{13}+\sigma_{46} \\
\sigma_{34}-\sigma_{16} & \sigma_{12}+\sigma_{45} & \sigma_{5}^{2}-\sigma_{1}^{2}-\sigma_{3}^{2} & \sigma_{23}+\sigma_{56} \\
\sigma_{15}-\sigma_{24} & \sigma_{13}+\sigma_{46} & \sigma_{23}+\sigma_{56} & \sigma_{6}^{2}-\sigma_{1}^{2}-\sigma_{2}^{2}
\end{array}\right] \\
\mathbf{L}_{\mathrm{m}}(z)=z \times\left[\begin{array}{cccc}
\mu_{0}+\sigma_{0}^{2} & \mu_{4}+2 \sigma_{04} & \mu_{5}+2 \sigma_{05} & \mu_{6}+2 \sigma_{06} \\
\mu_{4}+2 \sigma_{04} & \mu_{0}+\sigma_{0}^{2} & -\mu_{3}-2 \sigma_{03} & \mu_{2}+2 \sigma_{02} \\
\mu_{5}+2 \sigma_{05} & \mu_{3}+2 \sigma_{03} & \mu_{0}+\sigma_{0}^{2} & -\mu_{1}-2 \sigma_{01} \\
\mu_{6}+2 \sigma_{06} & -\mu_{2}-2 \sigma_{02} & \mu_{1}+2 \sigma_{01} & \mu_{0}+\sigma_{0}^{2}
\end{array}\right]
\end{gathered}
$$

It is worth noticing that Eq. (30) is exactly the expression we already derived in Eq. (26) of [9] where we extend the scope of the depolarizing Mueller calculus to parallel that established by Jones for his calculus based on the layered-medium interpretation. Eq. (31) is slightly different from that obtained in [9] where a particular order for different elementary layers is assumed. This order produces a symmetry breaking in the model and leads to a necessary consideration of the noncommutativity property of $\mathbf{G}_{\mathrm{i}}$. It is stated in [9] that the expression of the non-depolarizing part is dependent on the order of $\mathbf{G}_{\mathrm{i}}$ while the depolarizing part is not. However, two configurations of this depolarizing part differ only by the signs of the covariance terms since the commutators are antisymmetric $\left(\left[\mathbf{G}_{i}, \mathbf{G}_{\mathrm{j}}\right]=-\left[\mathbf{G}_{\mathrm{j}}, \mathbf{G}_{\mathrm{i}}\right]\right)$. It is possible to eliminate this difficulty with the order of the $\mathbf{G}_{\mathrm{i}}$ by assuming a random permutation of the order of the layers and by averaging all the differential matrices resulting from these permutations. Since the depolarizing part is order independent its average is always the solution given by Eq. (30). For the depolarizing part, each entry of the matrix is determined by two couples of indices $i j$ and $k l$ corresponding to the covariance terms between two elementary properties (see Eq. (27) in [9]). The set of all the permutations can thus be described by $\{(\mathrm{i}, \mathrm{j})(\mathrm{k}, \mathrm{l})$; (i,j) $(\mathrm{l}, \mathrm{k})$; (j,i) $(\mathrm{k}, \mathrm{l})$; (j,i) $(\mathrm{l}, \mathrm{k})\}$. Given the antisymmetric property of the commutators, it is obvious that all these terms disappear when averaging and the expression of the non-depolarizing part obtained in this case is exactly the expression given in Eq. (31).

In summary, the model based on the notion of stacking thin layers with random width associated with each of the elementary optical properties is directly related to the Gaussian white noise assumption. This provides a solution that is independent of the order of the layer elements and therefore generalizes the results given in [9] by solving the problem of non-commutativity of the previous model.

\section{DISCUSSION.}

We will now discuss the various assumptions that have been made in the preceding paragraphs as well as some results found in recent publications on the subject. Some results in the literature [10] assumed that their results are only valid as a first approximation (in fact keeping terms to the first order in $z$ only). But, what are we talking about when we are talking about first order in $z$ approximation from an experimental point of view?

The aim is then to define the conditions for the experimental use of all these theoretical results.

The first hypothesis is that of Gaussian fluctuations. This assumption implies that the fluctuations result in a very large number of elementary random effects. The result of these effects then follows a Gaussian law in virtue of the well known central limit theorem. It is, therefore natural without other information to assume that the fluctuations are Gaussian.

The second hypothesis of finite horizon correlation condition which is critical to have a linear variation on a large scale associated to the hypothesis $\alpha . \zeta \ll<1$ where $\alpha$ formally denotes the amplitude of the fluctuations seems to be less intuitive. $\mathbf{m}_{\mathrm{ij}}(z)$ in Eq. (24) or $p_{\mathrm{i}}(z)$ in Eq.(26) are physical quantities which correspond with the true microscopic effects acting on a light beam (more precisely on the Stokes parameters associated to this beam). From a point of view which considers thickness of the medium on a much longer scale (macroscopic scale) than the scale (microscopic scale) determined by the correlation distance, the true fluctuations may be replaced by these quantities. By assuming that Eq. (24) or Eq. (26) are verified, $\mathbf{m}_{\mathrm{ij}}(z)$ or $p_{\mathrm{i}}(z)$ respectively are assumed to be purely random fluctuations which means that microscopic correlation length $\zeta$ is insignificant on a macroscopic scale determined by $z$, that is to say on the thickness scale of the sample under experiment. In this way, this 
hypothesis can be used to describe any situation involving two distinct length scales: one for microscopic effects and one for a macroscopic analysis. Since in most cases, the thickness of experimental samples is greater than the correlation length of the microscopic effects, it can be said that this assumption is in fact the most common from an experimental point of view and $\mathbf{L}(z)$ is well described by Eq. (27) when $z \gg \zeta$. The necessary hypothesis $\alpha . \zeta<<1$ states that it is not possible to apply this Eq. (27) if the amplitudes of the fluctuations are not small with respect to $(\zeta)^{-1}$.

We can therefore conclude that the linear solution described by Eq. (27) is the one that is the most common from an experimental point of view as it does not consider samples too thin.

The statistical properties of such differential Mueller matrices (especially the depolarizing part $\mathbf{L}_{\mathrm{u}}(\mathrm{z})$ ) can be analyzed [10] in terms of their equivalent reduced coherency matrices termed $\mathbf{C} 3$. In their paper the authors derive an expression of the depolarizing differential matrix and note that these results are only valid as a first approximation (in fact keeping terms to the first order in $z$ only).

Although these results are derived from a very different approach, they correspond to $z$ second order expansion of Eq. (3). Unlike the methods of cumulants which is an expansion in powers of $(\alpha . \zeta)$ as mentioned above, these authors use an expansion in successive power of $(\alpha . z)$ therefore limited to small $z$ values. Using this expansion in power of $(\alpha . z)$ of the exponential for small $z$ value leads to:

$$
\begin{aligned}
\mathbf{M}(z) & =\mathrm{I}+\left\langle\underset{\leftarrow}{\mathrm{Z}}\left[\left(\int_{0}^{\mathrm{z}} \mathbf{m}_{\mathrm{ND}}(\mathrm{s}) \mathrm{ds}\right)\right]\right\rangle+\frac{1}{2 !}\left\langle\underset{\leftarrow}{\mathrm{Z}}\left[\left(\int_{0}^{\mathrm{z}} \mathbf{m}_{\mathrm{ND}}(\mathrm{s}) \mathrm{ds}\right)^{2}\right]\right\rangle \\
& =\mathrm{I}+\int_{0}^{\mathrm{z}} \mathrm{dz}_{1}\left\langle\mathbf{m}_{\mathrm{ND}}\left(z_{1}\right)\right\rangle+\int_{0}^{\mathrm{z}} \mathrm{dz}_{2} \int_{0}^{\mathrm{z}_{2}} \mathrm{dz} \mathrm{z}_{1}\left\langle\mathbf{m}_{\mathrm{ND}}\left(z_{2}\right) \cdot \mathbf{m}_{\mathrm{ND}}\left(z_{1}\right)\right\rangle
\end{aligned}
$$

Eq. (21) is used to transform this matrix relationship in a set of scalar relationship of the form:

$$
1+\int_{0}^{\mathrm{z}} \mathrm{dz} \mathrm{z}_{1}\left\langle p_{i}\left(z_{1}\right)\right\rangle+\int_{0}^{\mathrm{z}} \mathrm{dz} \int_{0}^{\mathrm{z}_{2}} \mathrm{dz} \mathrm{z}_{1}\left\langle p_{i}\left(z_{\mathbf{2}}\right) \cdot p_{j}\left(z_{1}\right)\right\rangle
$$

The $z$ first order term of expression (33) leads obviously to the z-linear solution of the non-depolarizing part of $\mathbf{L}(z)$ when a homogeneous hypothesis is assumed. The second order term is the correlation one. Under the assumption of stationary processes, $\rho_{\mathrm{ij}}\left(z_{1}, z_{2}\right)=\left\langle p_{i}\left(z_{2}\right) \cdot p_{j}\left(z_{1}\right)\right\rangle$ the correlation between $p_{\mathrm{i}}\left(z_{2}\right)$ and $p_{\mathrm{j}}\left(z_{1}\right)$ is only a function of $\left(z_{2}-z_{1}\right)$. If this correlation is an analytic function, its first order expansion relatively to $\left(z_{2}-z_{1}\right)$ is given by $\left(z_{2}-z_{1}\right.$ is small since $z_{1}$ and $z_{2}$ are small):

$$
\rho_{\mathrm{ij}}\left(z_{1}, z_{2}\right)=\rho_{\mathrm{ij}}\left(z_{1}-z_{2}\right)=\rho_{\mathrm{ij}}(0)+\left.\frac{1}{2 !} \frac{\mathrm{d} \rho_{\mathrm{ij}}\left(z_{1}-z_{2}\right)}{\mathrm{d}\left(z_{1}-z_{2}\right)}\right|_{z_{1}=z_{2}}\left(z_{1}-z_{2}\right)+\mathrm{o}\left[\left(z_{1}-z_{2}\right)^{2}\right]
$$

Keeping a consistent notation that is used to describe the white noise solution leads to $\rho_{\mathrm{ij}}(0)=\mathrm{r}_{\mathrm{ij}}=\sigma_{\mathrm{ij}}-\mu_{\mathrm{i}} \mu_{\mathrm{j}}$ and we may write:

$$
\rho_{\mathrm{ij}}\left(z_{1}-z_{2}\right)=\mathrm{r}_{\mathrm{ij}}+\frac{1}{2 !} \Delta \mathrm{r}_{\mathrm{ij}}\left(z_{1}-z_{2}\right)+\mathrm{o}\left[\left(z_{1}-z_{2}\right)^{2}\right]
$$

If using this relationship in Eq. (33) we find:

$$
\int_{0}^{z} \mathrm{dz}_{1} \int_{0}^{z_{1}} \mathrm{dz}_{2}\left\langle p_{i}\left(z_{1}\right) \cdot p_{j}\left(z_{2}\right)\right\rangle=z^{2} \frac{\mathrm{r}_{\mathrm{ij}}}{2 !}+z^{3} \frac{\Delta \mathrm{r}_{\mathrm{ij}}}{3 !}
$$


It is interesting to note that the higher order terms of the development of Eq. (32) do not give any $z^{2}$ contributions even if we limit the development of the correlation function to its zero order term. This is not the case for the term in $z^{3}$ since a contribution can also come from the 3rd order term of Eq. (32), and so on for the $z$ higher order terms.

This result clearly shows that a $z$-quadratic solution for depolarizing part of the differential matrix is obtained if we assume an approximation to the zero order of the correlation functions of the processes that are supposed stationary. It means that the correlation is assumed to be locally constant. This is the underlying assumption to the results given in [10]. It is worth noticing that no assumption of low correlation length is used or necessary. However, it would be wrong to believe that this result can be applied to any correlation distance $\zeta$ since a sufficient decay has to be ensured by the condition $\alpha . z<<1$ in order to have vanishing higher orders.

It is now clear that this first order in $z$ solution is valid only for a sample thickness on which the correlation function can be considered as constant $(z<\zeta)$ and will be valid on a much greater length than the amplitude of the fluctuations will be low $(\alpha . z<<1)$.

These hypothesis are valid for instance for very thin samples (microscopic scale) which is not the case for most of the test samples treated in the literature or for or for thicker samples but with very small fluctuations (thus a very low depolarization) and as long as it can be considered that the correlation is constant.

How the polarization properties and the depolarization ones are growing up linearly or quadratically with the thickness of the medium are clearly explained and the experimental conditions of application are also well defined.

Now consider the equivalent reduced coherency matrices termed $\mathbf{C} 3$ defined [7] by its entries expressed through those of $\mathbf{L}_{\mathrm{u}}(z)$ by the following relations:

$$
\begin{aligned}
& \mathbf{C} 3_{11}=\frac{1}{4}\left(\mathbf{L}_{u 11}+\mathbf{L}_{u 22}-\mathbf{L}_{u 33}-\mathbf{L}_{u 44}\right) \\
& \mathbf{C} 3_{22}=\frac{1}{4}\left(\mathbf{L}_{u 11}-\mathbf{L}_{u 22}+\mathbf{L}_{u 33}-\mathbf{L}_{u 44}\right) \\
& \mathbf{C} 3_{33}=\frac{1}{4}\left(\mathbf{L}_{u 11}-\mathbf{L}_{u 22}-\mathbf{L}_{u 33}+\mathbf{L}_{u 44}\right) \\
& \mathbf{C} 3_{12}=\mathbf{C} 3_{21}^{*}=\frac{1}{2}\left(\mathbf{L}_{u 23}+i \mathbf{L}_{u 14}\right) \\
& \mathbf{C} 3_{13}=\mathbf{C} 3_{31}^{*}=\frac{1}{2}\left(\mathbf{L}_{u 24}-i \mathbf{L}_{u 13}\right) \\
& \mathbf{C} 3_{23}=\mathbf{C} 3_{32}^{*}=\frac{1}{2}\left(\mathbf{L}_{u 34}+i \mathbf{L}_{u 12}\right)
\end{aligned}
$$

If $\sigma(\mathrm{AB})$ is the covariance of $\mathrm{AB}$ defined by the average value of $(\mathrm{A}-\langle\mathrm{A}\rangle)$. $(\mathrm{B}-\langle\mathrm{B}\rangle)^{*}, \mathrm{~L}, \mathrm{~L}$, and $\mathrm{C}$ are defined in the spectroscopic notation by $\mathrm{L}=-\left(p_{1+} \mathrm{i} . p_{4}\right)$ with $\langle\mathrm{L}\rangle=-\left(\mu_{1}+\mathrm{i} . \mu_{4}\right), \mathrm{L}^{\prime}=-\left(p_{2}+\mathrm{i} . p_{5}\right), \mathrm{C}=\left(p_{3}+\mathrm{i} . p_{6}\right)$ with the corresponding average values, Eq. (30) gives straightforwardly:

$$
\mathbf{C 3}\left(\mathbf{L}_{\mathrm{u}}(z)\right)=\frac{z}{2} \times\left[\begin{array}{ccc}
\sigma\left(|\mathrm{L}|^{2}\right) & \sigma\left(\mathrm{LL}^{*}\right) & \sigma\left(\mathrm{LC}^{*}\right) \\
\sigma\left(\mathrm{L}^{*} \mathrm{~L}^{\prime}\right) & \sigma\left(\left|\mathrm{L}^{\prime}\right|^{2}\right) & \sigma\left(\mathrm{LC}^{*}\right) \\
\sigma\left(\mathrm{L}^{*} \mathrm{C}\right) & \sigma\left(\mathrm{L}^{* *} \mathrm{C}\right) & \sigma\left(|\mathrm{C}|^{2}\right)
\end{array}\right]
$$

With the exception of the notation change, this result is directly related to Eq. (9) of [10]. However, different comments should be stated. Unlike [10] no assumption of first order approximation is required to obtain Eq. (38) when the random processes are Gaussian white noise-like since in this latter case this is an exact $z$-linear solution. The second point concerns the $z$-dependence of the solution and the possibility of having a $z$-linear variation for homogeneous media as we demonstrated. From the point of view of applications, this result and the knowledge of its terms of use, greatly enhances the utility of the method proposed by [10]. 


\section{CONCLUSION}

In summary, we show that assumption of short correlation distances is sufficient to ensure the existence of homogeneous depolarizing medium meaning the linearity of $\log (\mathbf{M}(z))$ according to $z$. In the limit of very small values of the product of amplitude of random fluctuations and correlation distance, only the first terms of the expansion survives and the expression of $\mathbf{M}(z)$ is the same as obtained under a white noise assumption. We show that under this white noise and second order stationary processes assumption, it is possible to derive the existence of a solution of depolarizing differential Mueller matrix as a mix of non-depolarizing media. It is worth noticing that in order for $\mathbf{M}$ to become homogeneous the homogeneity of the underlying non-depolarizing matrices has to be sacrificed. The random fluctuations make the underlying non-depolarizing matrices locally non-homogeneous even if the average behavior remains homogeneous.

Such a medium is thus characterized by linear changes of its differential optical properties with $z$ the thickness of the medium. We clarify this solution in the particular case where the random fluctuations processes associated to the optical properties are Gaussian white noise-like. Moreover, the solution obtained in the case is directly related to the physical model that we have recently proposed [9]. This model is based on the notion of stacking infinitely thin layers associated with each of the elementary optical properties introduced by Jones [1]. It assumes that the thickness of the layer elements varies randomly. A solution to the problem of non-commutativity of our previously proposed model is given by assuming a random permutation of the order of the layers and by averaging all the differential matrices resulting from these permutations. It is shown that the underlying assumption in this case is the Gaussian white noise assumption. Eventually we demonstrate in what condition the polarization properties and the depolarization ones are growing up linearly or quadratically with the thickness of the medium. The experimental conditions for applying such results are also well defined. We also demonstrate that for Gaussian white noise fluctuations of the optical properties the hypothesis of first order variations in $z$ is no longer necessary to analyse the statistical properties of the depolarizing part of the differential Mueller matrix by using the approach proposed recently in [10]. This result makes this method of analysis even more interesting to describe the fluctuation in optical properties of such a material. The conditions for using this method in other cases are also specified.

\section{APPENDIX A}

From Eq. (23) and Eq. (21) the integral of the first and second z-ordered cumulants are given by:

$$
\begin{aligned}
\int_{0}^{z} \mathbf{c}^{(\mathbf{1})}(s) \mathrm{d} s= & \int_{0}^{z}\langle\mathbf{m}(s)\rangle \mathrm{d} s=\sum_{\mathrm{i}=0}^{6} \int_{0}^{z} \mu_{\mathrm{i}} \mathrm{G}_{\mathrm{i}} \mathrm{d} s=z \sum_{\mathrm{i}=0}^{6} \mu_{\mathrm{i}} \mathbf{G}_{\mathrm{i}} \\
\int_{0}^{z} \mathbf{c}^{(2)}(s) \mathrm{d} s= & \int_{0}^{z}\left\langle\mathbf{m}(s)^{2}\right\rangle \mathrm{d} s-\frac{1}{2} \mathrm{Z}\left[\left(\int_{0}^{z}\langle\mathbf{m}(s)\rangle \mathrm{d} s\right)^{2}\right] \\
= & \sum_{\mathrm{i}, \mathrm{j}=0}^{6}\left(\int_{0}^{\mathrm{z}} \mathrm{dz}_{2} \int_{0}^{\mathrm{z}_{2}} \mathrm{dz} \mathrm{z}_{1}\left\langle\left(p_{\mathrm{i}}\left(\mathrm{z}_{2}\right) \mathbf{G}_{\mathrm{i}}\right)\left(p_{\mathrm{j}}\left(\mathrm{z}_{1}\right) \mathbf{G}_{\mathrm{j}}\right)\right\rangle\right) \\
& -\frac{1}{2} \sum_{\mathrm{i}, \mathrm{j}=0}^{6} 2\left(\int_{0}^{\mathrm{z}} \mathrm{dz}_{2} \int_{0}^{\mathrm{z}_{2}} \mathrm{dz} \mathrm{z}_{1}\left\langle p_{\mathrm{i}}\left(\mathrm{z}_{2}\right) \mathbf{G}_{\mathrm{i}}\right\rangle\left\langle p_{\mathrm{j}}\left(\mathrm{z}_{1}\right) \mathbf{G}_{\mathrm{j}}\right\rangle\right) \\
= & \sum_{\mathrm{i}, \mathrm{j}=0}^{6}\left(\mathrm{G}_{\mathrm{i}} \mathrm{G}_{\mathrm{j}} \int_{0}^{\mathrm{z}} \mathrm{dz}_{2} \int_{0}^{\mathrm{z}_{2}} \mathrm{dz} \mathrm{z}_{1}\left(\left\langle\mathrm{p}_{\mathrm{i}}\left(\mathrm{z}_{2}\right) \mathrm{p}_{\mathrm{j}}\left(\mathrm{z}_{1}\right)\right\rangle-\mu_{\mathrm{i}} \mu_{\mathrm{j}}\right)\right)
\end{aligned}
$$

Under the hypothesis of uncorrelated fluctuations of the processes given by Eq.(26): 


$$
\begin{aligned}
\int_{0}^{z} \mathbf{c}^{(2)}(s) \mathrm{d} s & =\sum_{\mathrm{i}, \mathrm{j}=0}^{6}\left(\mathrm{G}_{\mathrm{i}} \mathrm{G}_{\mathrm{j}} \int_{0}^{\mathrm{z}} \mathrm{d} \mathrm{z}_{2} \int_{0}^{\mathrm{z}_{2}} \mathrm{dz} \mathrm{z}_{1} \sigma_{\mathrm{ij}} \delta\left(\mathrm{z}_{2}-\mathrm{z}_{1}\right)\right) \\
& =z \sum_{\mathrm{i}, \mathrm{j}=0}^{6}\left(\sigma_{\mathrm{ij}} \mathrm{G}_{\mathrm{i}} \mathrm{G}_{\mathrm{j}}\right)
\end{aligned}
$$

From Eq.(25), Eq. (A1) and Eq. (A2) $\left\langle\mathbf{M}_{\mathbf{n d}}(z)>\right.$ is thus reduced to the expression of Eq. (27).

\section{References}

1. R.C. Jones, "A new calculus for the treatement of optical systems. VII properties of the N-matrices," J. Opt. Soc. Am. 38, 671-685 (1948)

2. R. M. A. Azzam, "Propagation of partially polarized light through anisotropic media with or without depolarization: A differential 4x4 matrix calculus," J. Opt. Soc. Am. 68, 1756-1767 (1978)

3. R. Ossikovski, "Differential matrix formalism for depolarizing anisotropic media," Opt. Letters. 36, 2330-2332 (2011)

4. N. Ortega-Quijano, J.L. Arce-Diego, "Mueller matrix differential decomposition," Opt. Letters 36, 1942-1944 (2011).

5. N. Ortega-Quijano, J.L. Arce-Diego, "Depolarizing differential Mueller matrices," Opt. Letters 36, 2429-2431 (2011).

6. Felix Gantmacher, Theory of matrices (AMS Chelsea publishing, 1959)

7. R. Ossikovski, V. Devlaminck, "General criterion for the physical realizability of the differential Mueller matrix," Opt. Lett. 39, 1216-1219 (2014)

8. V. Devlaminck and R. Ossikovski, "Uniqueness of the differential Mueller matrix of uniform homogeneous media," Opt. Lett. 39, 3149-3152 (2014).

9. V. Devlaminck, "Physical model of differential Mueller matrix for depolarizing uniform media," J. Opt. Soc. Am. 30, 2196-2204 (2013)

10. R. Ossikovski, O. Arteaga, "Statistical meaning of the differential Mueller matrix of depolarizing homogeneous media," Opt. Lett. 39, 4470-4473 (2014)

11. R. Barakat, "Exponential versions of the Jones and Mueller-Jones polarization matrices," J. Opt. Soc. Am. A 13, 158163 (1996)

12. K. Kim, L. Mandel, and E. Wolf, "Relationship between Jones and Mueller matrices for random media," J. Opt. Soc. Am. A 4, 433-437 (1987)

13. J.W. Goodman, Statistical Optics (John Winley \&Son, 1985)

14. Kendall, M.G., Stuart, A., The Advanced Theory of Statistics, Vol. 1 (3rd Edition), (Griffin, London, 1969)

15. R.F. Fox, "Critique of the generalized cumulant expansion method," J. Math. Phy. 17, 1148-1153 (1976)

16. N.G. van Kampen, Stochastic processes in physics and chemistry (North Holland Publishing Company, 1981)

17. R.F. Fox, "Gaussian stochastic processes in physics," Phy. Rep. 48, 179-283 (1978) 